РАЗДЕЛ V. МАТЕМАТИКА

\title{
ZHukenov D.Z. \\ A method for determining the simplicity of a given number
}

doi: 10.18411/scienceconf-03-2021-22

idsp: scienceconf-03-2021-22

\section{Abstract}

Finding primes is interesting not only from a theoretical point of view. It allows you to improve calculation methods, test computers. In addition, prime number theory is used in cryptography to develop ciphers. The method for finding prime numbers, based on the Erotosthenes sieve, involves dividing a given number $\mathrm{x}$ by all primes and therefore it is not practical. The method I propose allows you to determine the simplicity of a given number, i.e. numbers of the form $6 n+1$ and $6 n-1$, where $n$ - natural number method for determining the simplicity of a given number. annotation Finding primes is interesting not only from a theoretical point of view. It allows you to improve calculation methods, test computers. In addition, prime number theory is used in cryptography to develop ciphers. The method for finding prime numbers, based on the Erotosthenes sieve, involves dividing a given number $\mathrm{x}$ by all primes and therefore it is not practical. The method I propose allows you to determine the simplicity of a given number, i.e. numbers of the form $6 n+1$ and $6 n-1$, where $n$ - natural number.

Key words: prime numbers, complex numbers, quadratic equation, remainder.

Until now, a simple regularity of the distribution of primes has not been established, there is no effective method for determining the prime number, there is no satisfactory formula for the number of prime numbers, and in general, the amount of knowledge about the properties, signs, and behavior of prime numbers is very scarce and therefore there is no complete picture of this phenomenon. This is primarily due to their exceptional complexity. The method based on the Erotosthenes sieve [1,370] involves dividing a given number $\mathrm{x}$ into all primes and therefore it is not practical. The problems of the distribution of paired primes have not been solved $[2,10]$ It is known that prime numbers can be represented in the form $6 \mathrm{n}$ +1 or $6 n-1$, where $n$ is a natural number, but among these numbers there can be complex number. The method I propose allows you to determine the simplicity of any a given number of this kind. It is easy to prove that the product of two such numbers also has this form. for example $(6 \mathrm{i}+1)(6 \mathrm{j}+1)=36 \mathrm{ij}+6(\mathrm{i}+\mathrm{j})+1(6 \mathrm{i}-1)(6 \mathrm{j}-1)=36 \mathrm{ij}-6(\mathrm{i}+\mathrm{j})+1(6 \mathrm{i}-1)(6 \mathrm{j}+1)=$ 36ij-6 (i-j) -1 where $i, j$ are natural numbers. If we subtract or add 1 , then we divide by 6 , we get a number of the form $6 i j+(i+j)$, where $(i+j)$ is the remainder of the number when divided by 6 . Consider specific examples. For example, given the number 301 . It is necessary to determine whether it is prime or not. Subtract 1 and dividing by 6 we get $50=6 * 10-1050$ $=6 * 9-450=6 * 8+250=6 * 7+850=6 * 6+14$ residues are separated from each other by 6 . Remains can be produced keeping such numbers: For example $(8=1+7=2+6=3+5$ $=4+4) 1 * 7.2 * 6.3 * 5.4 * 4$ or $1 *(8-1), 2 *(8-2), 3 *(8-3), 4 *(8-4)$ or $1 * 8-1 * 1,2 * 8$ $2 * 2=-2^{2}+2 * 8=-\left(2^{2}-2 * 8\right)$, The remainder $10=(1+9.2+8.3+7.4+6.5+5)$ can be as the product $1 * 9.2 * 8.3 * 7,4 * 6$, or $5 * 5$, i.e. varies from 9 to 25 . The remainder is 4 as $1 *$ $3.2 * 2$. The remainder is $2-1 * 1$. Let us write in what limits the products of numbers that make up the amount of balances. $6 * 10-10$ from 9 to $256 * 9-4$ from 3 to $46 * 8+216 * 7$ +8 from 7 to $126 * 6+14$ from 13 to 49 We have to solve a quadratic equation. For example, the remainder of 8 can be represented by $\mathrm{x} *(8-\mathrm{x})=8 \mathrm{x}-\mathrm{x}^{2}$ and must be equal to a factor of 6 , for example 7 . The radical expression $8^{2}-4 * 7$ should be the square of the number. In our case the root is 6 . The roots of the equation are 7 and $1.6 * 7+8=6 * 7+1+7$ i.e. 
$301=(6 * 1+1)(6 * 7+1)=7 * 43$ Check the remainder of $10.10^{2}-4 * 10=60$ is not the square of a number. Therefore, the number 301 is difficult. Consider the number 643. (643-1) / $6=107.107=6 * 18-1$ Therefore $6 * 20-1312,22,30 \ldots 426 * 19-76,10,126 * 18-116$ $* 17+54,66 * 16+1110,18 \ldots 306 * 15+1716, \ldots 72$ No residual decomposition is equal to a factor of 6 , therefore our number 643 is prime. Consider the number $23 .(23+1) / 6$ $=4.4=6 * 1-26 * 2-89-1,10-2, \ldots 6 * 1-23-1,4-2, \ldots 6 * 0+45-1,6-2, \ldots$ Here the remainder should be expressed as the difference of two numbers. The expansion of the remainders does not equal a factor of six, so 23 is prime. Further expansion of the number is meaningless.

$$
* * *
$$

1. Kulikov L. Ya. Algebra and number theory. M. "High School" .1979

2. Ayerland M. Rosen Classical introduction to modern number theory. Moscow. "Peace" 1987 